\title{
Liquid-Liquid Equilibria for the System Perfluorodecalin/1-Heptene/n-Heptane/n-Hexane
}

\author{
Gabriela Bernardo-GII* \\ Departamento Engenharia Quimica, Instituto Superior Técnico, 1096 Lisboa codex, Portugal
}

Luis J. S. Soares

Universidade do Minho, L. Paço, 4719 Braga codex, Portugal

\begin{abstract}
Liquid-liquid equillibrla for the quaternary system perfluorodecalln (PFD)/1-heptene/n-heptane/n-hexane at 288.15 K (type III) and 298.15 K (type II) and for the ternary systems PFD/1-heptene/n-heptane, PFD/1-heptene/n-hexane, and PFD/n-heptane/n-hexane at the same temperatures are reported. The experimental results are compared with values predicted by using the NRTL, the UNIQUAC, and the UNIFAC models.
\end{abstract}

\section{Introduction}

Multicomponent mixtures containing perfluorocarbons and hydrocarbons change swiftly from type to type pattern over a small temperature range.

After the experimental determination of binary solubility data for some perfluorodecalin/hydrocarbon systems (1), this paper reports the results of measurements on the liquid-liquid equilibrium for quaternary and ternary systems containing perfluorodecalin, 1-heptene, $n$-heptane, and $n$-hexane.

Predictions of equilibrium data for these mixtures were made by using the NRTL and UNIQUAC perfluorodecalin/hydrocarbon parameters obtained from binary data. Hydrocarbon/hydrocarbon parameters were calculated by correlating the ternary data. The results are compared with the experimental ones.

The same calculations were made by using the UNIFAC model. The $\mathrm{CH}_{3} / \mathrm{CF}_{2}$ (c) and $\mathrm{CH}_{2} \mathrm{CH} / \mathrm{CF}_{2}$ (c) parameters were obtained from binary data $(1,6)$.

\section{Experimental Section}

The experimental work was carried out by using a thermostated miniature cell similar to that described by Soares et al.

Table I. Experimental Liquid-Liquid Equilibrium Compositions for the Ternary System PFD (1)/1-Heptene (2)/n-Heptane (3)

\begin{tabular}{ccccccc}
\hline & \multicolumn{6}{c}{ weight fractions } \\
\cline { 2 - 7 }$T, \mathrm{~K}$ & $X_{11}$ & $X_{21}$ & $X_{31}$ & $X_{12}$ & $X_{22}$ & $X_{32}$ \\
\hline 288.15 & 0.2503 & 0.1220 & 0.6277 & 0.9489 & 0.0080 & 0.0431 \\
& 0.2410 & 0.2070 & 0.5520 & 0.9487 & 0.0139 & 0.0374 \\
& 0.2356 & 0.3131 & 0.4513 & 0.9489 & 0.0210 & 0.0301 \\
& 0.2256 & 0.3924 & 0.3820 & 0.9490 & 0.0264 & 0.0246 \\
& 0.2228 & 0.4439 & 0.3333 & 0.9490 & 0.0293 & 0.0217 \\
& 0.2192 & 0.4907 & 0.2901 & 0.9491 & 0.0326 & 0.0183 \\
& 0.2154 & 0.5206 & 0.2640 & 0.9492 & 0.0339 & 0.0169 \\
& 0.2151 & 0.5642 & 0.2207 & 0.9491 & 0.0381 & 0.0128 \\
& 0.2124 & 0.5852 & 0.2024 & 0.9492 & 0.0385 & 0.0123 \\
& 0.2078 & 0.6522 & 0.1400 & 0.9494 & 0.0425 & 0.0081 \\
298.15 & 0.2928 & 0.5448 & 0.1624 & 0.9306 & 0.0548 & 0.0146 \\
& 0.3018 & 0.4306 & 0.2676 & 0.9301 & 0.0449 & 0.0250 \\
& 0.3088 & 0.3237 & 0.3675 & 0.9299 & 0.0354 & 0.0347 \\
& 0.3162 & 0.2590 & 0.4248 & 0.9289 & 0.0292 & 0.0419 \\
& 0.3181 & 0.2202 & 0.4617 & 0.9282 & 0.0251 & 0.0467 \\
& 0.3235 & 0.1788 & 0.4977 & 0.9283 & 0.0191 & 0.0526 \\
& 0.3258 & 0.1508 & 0.5234 & 0.9281 & 0.0146 & 0.0573 \\
& 0.3262 & 0.1200 & 0.5538 & 0.9281 & 0.0138 & 0.0581
\end{tabular}

Table II. Experimental Liquid-Liquid Equilibrium Compositions for the Ternary System PFD (1)/1-Heptene (2)/n-Hexane (4)

\begin{tabular}{ccccccc}
\hline & \multicolumn{6}{c}{ weight fractions } \\
\cline { 2 - 7 }$T, \mathrm{~K}$ & $X_{11}$ & $X_{21}$ & $X_{41}$ & $X_{12}$ & $X_{22}$ & $X_{42}$ \\
\hline 288.15 & 0.3619 & 0.1582 & 0.4799 & 0.9160 & 0.0190 & 0.0650 \\
& 0.3309 & 0.2373 & 0.4318 & 0.9248 & 0.0258 & 0.0494 \\
& 0.3053 & 0.3091 & 0.3856 & 0.9327 & 0.0299 & 0.0374 \\
& 0.2826 & 0.3659 & 0.3515 & 0.9335 & 0.0335 & 0.0330 \\
& 0.2737 & 0.4063 & 0.3200 & 0.9337 & 0.0348 & 0.0315 \\
& 0.2548 & 0.4644 & 0.2808 & 0.9376 & 0.0372 & 0.0252 \\
& 0.2397 & 0.5294 & 0.2309 & 0.9378 & 0.0424 & 0.0198 \\
298.15 & 0.2315 & 0.5953 & 0.1732 & 0.9404 & 0.0441 & 0.0155 \\
& 0.3067 & 0.5570 & 0.1363 & 0.9257 & 0.0605 & 0.0138 \\
& 0.3584 & 0.3654 & 0.2762 & 0.9124 & 0.0475 & 0.0401 \\
& 0.4371 & 0.2303 & 0.3326 & 0.8971 & 0.0382 & 0.0647 \\
& 0.4942 & 0.1571 & 0.3487 & 0.8736 & 0.0368 & 0.0896 \\
& 0.4988 & 0.1506 & 0.3506 & 0.8698 & 0.0327 & 0.0975 \\
& 0.5535 & 0.0971 & 0.3494 & 0.8562 & 0.0288 & 0.1150
\end{tabular}

Table III. Experimental Liquid-Liquid Equilibrium Compositions for the Ternary System PFD (1)/n-Heptane (3)/n-Hexane (4)

\begin{tabular}{ccccccc}
\hline & \multicolumn{6}{c}{ weight fractions } \\
\cline { 2 - 7 }$T, \mathrm{~K}$ & $X_{11}$ & $X_{31}$ & $X_{41}$ & $X_{12}$ & $X_{32}$ & $X_{42}$ \\
\hline 288.15 & 0.4056 & 0.0581 & 0.5363 & 0.8989 & 0.0077 & 0.0934 \\
& 0.3864 & 0.1040 & 0.5096 & 0.9002 & 0.0135 & 0.0863 \\
& 0.3436 & 0.1930 & 0.4634 & 0.9117 & 0.0218 & 0.0665 \\
& 0.3218 & 0.2576 & 0.4206 & 0.9163 & 0.0273 & 0.0564 \\
& 0.3001 & 0.3300 & 0.3699 & 0.9206 & 0.0339 & 0.0455 \\
& 0.2820 & 0.3933 & 0.3247 & 0.9284 & 0.0362 & 0.0354 \\
& 0.2735 & 0.4572 & 0.2693 & 0.9324 & 0.0397 & 0.0279 \\
& 0.2646 & 0.5092 & 0.2262 & 0.9351 & 0.0432 & 0.0217 \\
& 0.2565 & 0.5603 & 0.1832 & 0.9404 & 0.0445 & 0.0151 \\
298.15 & 0.2562 & 0.6098 & 0.1340 & 0.9419 & 0.0469 & 0.0112 \\
& 0.9241 & 0.0647 & 0.0112 & 0.3759 & 0.5485 & 0.0756 \\
& 0.9234 & 0.0618 & 0.0148 & 0.3876 & 0.5004 & 0.1120 \\
& 0.9132 & 0.0565 & 0.0303 & 0.4157 & 0.4003 & 0.1840 \\
& 0.9030 & 0.0495 & 0.0475 & 0.4475 & 0.3125 & 0.2400 \\
& 0.8917 & 0.0435 & 0.0648 & 0.4886 & 0.2310 & 0.2804 \\
& 0.8756 & 0.0327 & 0.0857 & 0.5209 & 0.1654 & 0.3137 \\
& 0.8592 & 0.0352 & 0.1056 & 0.5566 & 0.1186 & 0.3248 \\
& 0.8485 & 0.0297 & 0.1218 & 0.5874 & 0.0875 & 0.3251
\end{tabular}

(2). Temperature was controlled within an accuracy of 0.01 $\mathrm{K}$ and was measured by a precision thermometer. The mixtures were stirred with a magnetic stirrer for at least $30 \mathrm{~min}$ and allowed to settle during a period of $4 \mathrm{~h}$. The tie lines were obtained by analyzing the compositions of the two conjugate phases in equilibrium by means of a gas chromatograph (Pye/Unicam) connected to an integrator (Varian CDS 111). Special care was taken with the tie-line determination above room temperature to avoid phase splitting due to cooling.

Calibration curves were obtained by using at least $10 \mathrm{mix}$ tures of known composition of which four to six chromatograms were obtained. Four to six samples of both phases were analyzed to minimize the experimental error. Weight fraction measurements were reproducible to within \pm 0.002 . 
Table IV. Experimental Liquid-Liquid Equilibrium Compositions for the Quaternary System PFD (1)/1-Heptene (2)/n-Heptane (3)/n-Hexane (4)

\begin{tabular}{|c|c|c|c|c|c|c|c|c|}
\hline \multirow[b]{2}{*}{$T, \mathrm{~K}$} & \multicolumn{8}{|c|}{ weight fractions } \\
\hline & $X_{11}$ & $X_{21}$ & $X_{31}$ & $X_{41}$ & $X_{12}$ & $X_{22}$ & $X_{32}$ & $X_{42}$ \\
\hline \multirow[t]{16}{*}{288.15} & 0.9422 & 0.0348 & 0.0078 & 0.0152 & 0.2469 & 0.4454 & 0.1127 & 0.1950 \\
\hline & 0.9423 & 0.0297 & 0.0158 & 0.0122 & 0.2548 & 0.3705 & 0.2213 & 0.1534 \\
\hline & 0.9447 & 0.0238 & 0.0238 & 0.0077 & 0.2452 & 0.2969 & 0.3485 & 0.1094 \\
\hline & 0.9445 & 0.0203 & 0.0288 & 0.0064 & 0.2558 & 0.2521 & 0.4086 & 0.0835 \\
\hline & 0.9459 & 0.0178 & 0.0316 & 0.0047 & 0.2628 & 0.2110 & 0.4632 & 0.0630 \\
\hline & 0.9429 & 0.0213 & 0.0289 & 0.0069 & 0.2640 & 0.2434 & 0.4056 & 0.0870 \\
\hline & 0.9458 & 0.0261 & 0.0188 & 0.0093 & 0.2490 & 0.3548 & 0.2806 & 0.1156 \\
\hline & 0.9374 & 0.0222 & 0.0139 & 0.0265 & 0.3064 & 0.2374 & 0.1724 & 0.2838 \\
\hline & 0.9326 & 0.0190 & 0.0107 & 0.0377 & 0.3119 & 0.1961 & 0.1277 & 0.3643 \\
\hline & 0.9278 & 0.0179 & 0.0093 & 0.0450 & 0.3465 & 0.1542 & 0.0926 & 0.4067 \\
\hline & 0.9329 & 0.0239 & 0.0073 & 0.0359 & 0.3086 & 0.2478 & 0.0903 & 0.3533 \\
\hline & 0.9352 & 0.0316 & 0.0055 & 0.0277 & 0.2885 & 0.3386 & 0.0738 & 0.2991 \\
\hline & 0.9395 & 0.0331 & 0.0042 & 0.0232 & 0.2773 & 0.3954 & 0.0567 & 0.2706 \\
\hline & 0.9411 & 0.0408 & 0.0029 & 0.0152 & 0.2609 & 0.5034 & 0.0441 & 0.1916 \\
\hline & 0.9455 & 0.0349 & 0.0098 & 0.0098 & 0.2536 & 0.4715 & 0.1441 & 0.1308 \\
\hline & 0.9428 & 0.0305 & 0.0191 & 0.0076 & 0.2594 & 0.3586 & 0.2798 & 0.1022 \\
\hline \multirow[t]{23}{*}{298.15} & 0.9243 & 0.0392 & 0.0212 & 0.0153 & 0.3434 & 0.3444 & 0.1917 & 0.1205 \\
\hline & 0.9146 & 0.0373 & 0.0197 & 0.0284 & 0.3722 & 0.2840 & 0.1552 & 0.1886 \\
\hline & 0.9046 & 0.0332 & 0.0176 & 0.0446 & 0.4108 & 0.2186 & 0.1169 & 0.2537 \\
\hline & 0.8963 & 0.0371 & 0.0151 & 0.0515 & 0.4392 & 0.1934 & 0.0952 & 0.2722 \\
\hline & 0.8787 & 0.0260 & 0.0137 & 0.0816 & 0.4906 & 0.1214 & 0.0655 & 0.3225 \\
\hline & 0.8705 & 0.0202 & 0.0103 & 0.0990 & 0.5309 & 0.0810 & 0.0423 & 0.3458 \\
\hline & 0.8684 & 0.0270 & 0.0090 & 0.0956 & 0.5157 & 0.0975 & 0.0377 & 0.3491 \\
\hline & 0.8863 & 0.0093 & 0.0422 & 0.0622 & 0.4612 & 0.0376 & 0.2167 & 0.2845 \\
\hline & 0.8811 & 0.0074 & 0.0351 & 0.0764 & 0.4986 & 0.0342 & 0.1583 & 0.3089 \\
\hline & 0.8846 & 0.0153 & 0.0293 & 0.0708 & 0.4810 & 0.0791 & 0.1399 & 0.3000 \\
\hline & 0.8916 & 0.0113 & 0.0277 & 0.0694 & 0.4802 & 0.0717 & 0.1403 & 0.3078 \\
\hline & 0.8706 & 0.0185 & 0.0203 & 0.0906 & 0.5259 & 0.0547 & 0.0834 & 0.3360 \\
\hline & 0.8625 & 0.0162 & 0.0196 & 0.1017 & 0.5443 & 0.0386 & 0.0726 & 0.3445 \\
\hline & 0.8653 & 0.0040 & 0.0331 & 0.0976 & 0.5408 & 0.0132 & 0.1194 & 0.3266 \\
\hline & 0.9087 & 0.0114 & 0.0441 & 0.0358 & 0.4221 & 0.0483 & 0.3071 & 0.2225 \\
\hline & 0.9102 & 0.0077 & 0.0605 & 0.0216 & 0.3932 & 0.0256 & 0.4470 & 0.1342 \\
\hline & 0.9072 & 0.0070 & 0.0561 & 0.0297 & 0.4080 & 0.0305 & 0.3879 & 0.1736 \\
\hline & 0.9018 & 0.0065 & 0.0528 & 0.0389 & 0.4204 & 0.0294 & 0.3347 & 0.2155 \\
\hline & 0.8978 & 0.0032 & 0.0516 & 0.0474 & 0.4476 & 0.0234 & 0.2950 & 0.2340 \\
\hline & 0.9365 & 0.0492 & 0.0130 & 0.0013 & 0.2938 & 0.5509 & 0.1441 & 0.0115 \\
\hline & 0.9312 & 0.0489 & 0.0130 & 0.0069 & 0.3059 & 0.4951 & 0.1333 & 0.0657 \\
\hline & 0.9154 & 0.0374 & 0.0310 & 0.0162 & 0.3576 & 0.2846 & 0.2417 & 0.1161 \\
\hline & 0.9175 & 0.0438 & 0.0112 & 0.0275 & 0.3677 & 0.3476 & 0.0938 & 0.1909 \\
\hline
\end{tabular}

PFD (Aldrich/Europe, cis + trans mixture, minimum purity $95 \%$ ), $n$-hexane and $n$-heptane (Merck, minimum purity $99 \%$ ), and 1-heptene (Fluka, minimum purity $95 \%$ ) were used as supplied.

Tables I-III summarize the experimental results obtained for the ternary systems. Quaternary tie-line data are presented in Table IV.

\section{Analysis of Results}

Prediction of ternary and quaternary liquid-liquid equilibrium data was realized by using the well-known NRTL (3), UNIQUAC (4), and UNIFAC (5) models. Agreement between calculated and experimental data is expressed in terms of composition root-mean-square deviations (rmsd):

$$
\text { rmsd }=\left[\frac{\sum_{k=1}^{M} \sum_{1=1 /=1}^{N} \sum_{j=1}^{2}\left(x_{i j k}^{\text {caled }}-x_{i j k}^{\text {expt }}\right)}{2 N(M-1)}\right]^{1 / 2}
$$

NRTL and UNIQUAC Equations. Binary NRTL and UNIQUAC parameters were determined by using two alternative approaches:

Mothod A. This approach is the direct correlation of the ternary data, with the number of adjusted parameters varying from two to six, keeping $\alpha_{i j}$ (NRTL equation) constant and equal to 0.3 for hydrocarbon/hydrocarbon systems, according to Renon and Prausnitz (3). The parameters for PFD/hydrocarbon binaries were the recommended values $(1,6)$.
Table V. Recommended Values for NRTL and UNIQUAC Parameters at 288.15 and $298.15 \mathrm{~K}$

\begin{tabular}{cccccccc}
\hline & & \multicolumn{3}{c}{ NRTL } & & \multicolumn{2}{c}{ UNIQUAC } \\
\cline { 3 - 5 } system & $T$, & & $b_{12}$, & $b_{21}$, & & $c_{12}$, & $c_{21}$, \\
PFD/n-hexane & 288.15 & 0.4 & 369.6 & 651.1 & & 152.8 & -11.85 \\
& 298.15 & 0.4 & 318.6 & 585.7 & & 162.3 & -26.64 \\
PFD/n-heptane & 288.15 & 0.3 & 337.6 & 678.7 & & 158.7 & -8.34 \\
& 289.15 & 0.3 & 306.6 & 640.3 & & 162.0 & -17.33 \\
PFD/1-heptene & 288.15 & 0.3 & 319.3 & 757.0 & & 150.4 & -6.41 \\
& 298.15 & 0.3 & 285.3 & 731.4 & & 149.9 & -0.925
\end{tabular}

Method B. For the PFD/hydrocarbon binaries, the linear temperature dependence of parameters, obtained from the correlation of binary solubility data $(1,6)$ was used; hydrocarbon/hydrocarbon parameters were calculated by adjusting ternary tie-line data.

Binary parameters from ternary data were calculated by using the Nelder-Mead method for the minimization of the following objective functions:

$$
\begin{gathered}
F_{1}=\sum_{k=1}^{M} \sum_{i=1}^{N}\left[\ln \left(\gamma_{i 1} / \gamma_{i 2}\right)-\ln \left(x_{i 2} / x_{i 1}\right)\right]_{k}^{2} \\
F_{2}=\sum_{k=1}^{M} \sum_{i=1 j=1}^{N} \sum_{j=1}^{2}\left(x_{i j k}^{\text {calcd }}-x_{i j k}^{\text {expt })^{2}}\right.
\end{gathered}
$$

with $3 M>L, M$ being the available number of tie lines, $N$ the number of components, and $L$ the number of parameters. 
Table VI. rmsd $\left(\times 10^{3}\right)$ between Calculated and Experimental Values

\begin{tabular}{|c|c|c|c|c|c|c|c|}
\hline \multirow[b]{2}{*}{ system } & \multirow[b]{2}{*}{$T, \mathrm{~K}$} & \multirow[b]{2}{*}{ type } & \multicolumn{2}{|c|}{$\mathrm{NRTL}^{a}$} & \multicolumn{2}{|c|}{$\mathrm{UNIQUAC}^{a}$} & \multirow[b]{2}{*}{ UNIFAC } \\
\hline & & & A & B & $\mathrm{A}$ & B & \\
\hline \multirow{4}{*}{ PFD $/ n$-heptane $/ n$-hexane } & 288.15 & II & 5.4 & 8.7 & 5.4 & 8.7 & 10.5 \\
\hline & 298.15 & I & 5.5 & 5.7 & 13.9 & 14.7 & 18.9 \\
\hline & 288.15 & II & 5.8 & 5.8 & 8.4 & 9.2 & 16.1 \\
\hline & 298.15 & I & 6.6 & 7.0 & 8.1 & 8.6 & 15.5 \\
\hline \multirow[t]{2}{*}{ PFD/1-heptene/n-heptane } & 288.15 & II & 1.4 & 1.4 & 1.4 & 1.5 & 17.6 \\
\hline & 298.15 & II & 3.0 & 3.9 & 3.4 & 3.9 & 14.4 \\
\hline \multirow[t]{2}{*}{ PFD/1-heptene/n-heptane/n-hexane } & 288.15 & III & 9.0 & 10.4 & 18.5 & 17.1 & 26.4 \\
\hline & 298.15 & II & 11.6 & 11.6 & 15.8 & 13.0 & 15.9 \\
\hline
\end{tabular}

${ }^{a} \mathrm{~A}$, rmsd obtained by using method A; B, rmsd obtained by using method B.

The NRTL and UNIQUAC parameters recommended for the PFD/hydrocarbon binaries are presented in Table $\mathrm{V}$.

Table VI shows the values of rmsd between experimental and calculated compositions.

UNIFAC Method. UNIFAC group parameters for the interactions $\mathrm{CH}_{2} / \mathrm{CF}_{2}(\mathrm{c})$ and $\mathrm{CH}_{2}=\mathrm{CH} / \mathrm{CF}_{2}$ were obtained from mutual solubility data $(1,6)$. We consider these parameters to be dependent on the temperature and the number of carbon atoms of the hydrocarbon. For example, in the mixture $\mathrm{PFD} / n$-heptane/n-hexane we have the interactions $\mathrm{CH}_{2} / \mathrm{CF}_{2}$ (c) (6) and $\mathrm{CH}_{2} / \mathrm{CF}_{2}(\mathrm{c})(7)$.

Table VI also includes the values of rmsd obtained by using the UNIFAC model.

\section{Conclusions}

The experimental results were correlated in terms of NRTL, UNIQUAC, and UNIFAC equations. These models represent the binodal curves and the tie lines correctly either for a type I system or for a type II.

With slightly less accuracy (relative to the results obtained from direct correlation of data of each individual ternary system), it is possible to obtain a set of NRTL and UNIQUAC parameters applicable to all multicomponent mixtures of PFD and hydrocarbons.

The overall results are better for the NRTL equation using the recommended values of $\alpha_{l}$, although the predicted multicomponent data are strongly dependent on the selected $\alpha_{i j}$ for the partially miscible binaries.

The rmsd values between experimental and calculated compositions are, in general, larger for the UNIFAC method than for the NRTL and UNIQUAC equations.

The correlation of UNIFAC group interaction parameters with the number of carbon atoms of the hydrocarbon improves the prediction of multicomponent data significantly and it is easy to apply.

\section{Acknowledgment}

We express our gratitude to the Instituto Nacional de Investigação Cientifica, which through the CPQ (Universidade Técnica de Lisboa) and CQPA (Universidade do Minho) supported this work. The computing facilities offered by CIIST are also gratefully acknowledged.

\section{Appendlx}

Equations giving the activity coefficients as a function of composition and temperature are here stated very briefly.

NRTL Equation (3).

$$
\ln \gamma_{l}=\frac{\sum_{j=1}^{N} \tau_{j} G_{j j} x_{j}}{\sum_{k=1}^{N} G_{k} x_{k}}+\sum_{j=1}^{N} \frac{x j G_{i j}}{\sum_{k=1}^{N} x_{k} G_{k j}}\left(\tau_{l j}-\frac{\sum_{k=1}^{N} x_{k} G_{k j} \tau_{k j}}{\sum_{k=1}^{N} G_{k j} x_{k}}\right)
$$

$$
\begin{gathered}
\tau_{l j}=\frac{\left(g_{i j}-g_{y j}\right)}{R T}=\frac{b_{i j}}{T} \quad g_{i j}=g_{j} \\
G_{l j}=\exp \left(-\alpha_{i j} \tau_{l j}\right) \quad \alpha_{l j}=\alpha_{j i}
\end{gathered}
$$

The NRTL parameters are $b_{\| j}, b_{\mu}$, and $\alpha_{i j}$.

UNIQUAC Equation (4). This model has a combinatorial contribution to the activity coefficient, due to differences in size and shape of the molecules, and a residual contribution, due to energetic interactions:

$$
\ln \gamma_{i}=\ln \gamma_{i} \text { (comb) }+\ln \gamma_{i} \text { (res) }
$$

$$
\begin{aligned}
& \ln \gamma_{1}(\mathrm{comb})=\ln \frac{\phi_{1}}{x_{i}}+\frac{z}{2} q_{i} \ln \frac{\vartheta_{l}}{\phi_{i}}+I_{i}-\frac{\phi_{i}}{x_{1 j}} \sum_{=1}^{N} x_{j} l_{j} \\
& \ln \gamma_{i} \text { (res) }=q_{1}\left[1-\ln \left(\sum_{j=1}^{N} \vartheta_{j} \tau_{l j}\right)-\sum_{j=1}^{N} \frac{\vartheta_{j} \tau_{l j}}{\sum_{k=1}^{N} \vartheta_{k} \tau_{k j}}\right] \\
& l_{i}=\frac{z}{2}\left(r_{i}-q_{l}\right)-\left(r_{1}-1\right) \quad z=10 \\
& \tau_{i j}=\exp \left[-\frac{u_{i j}-u_{i j}}{R T}\right]=\exp \left[-\frac{c_{i j}}{T}\right] \quad u_{i j}=u_{j}
\end{aligned}
$$

$r_{i}$ and $q_{i}$ are pure component parameters that are measures of the van der Waals volumes and surface areas of molecule $i$. They are calculated as the sum of the group-volume and the group-area parameters, $R_{k}$ and $Q_{k}$ :

$$
r_{i}=\sum_{k=1}^{N G} v_{k} R_{k} \quad q_{i}=\sum_{k=1}^{N G} v_{k} Q_{k}
$$

where $v_{k i}$ is the number of groups of type $k$ in molecule $i$ and $N G$ is the total number of groups. Group parameters $R_{k}$ and $Q_{k}$ are obtained from van der Waals group volumes and surface areas, $V_{k}$ and $A_{k}$, given by Bondi $(7)$ :

$$
\begin{gathered}
R_{k}=V_{k} / 15.17 \quad Q_{k}=A_{k} /\left(2.5 \times 10^{9}\right) \\
\vartheta_{i}=\frac{x q_{l}}{\sum_{j=1}^{N} x q_{j}} \\
\phi_{l}=\frac{x_{i} r_{i}}{\sum_{j=1}^{N} x_{j} r_{j}}
\end{gathered}
$$

$c_{l /}$ and $c_{j}$ are UNIQUAC parameters.

UNIFAC Equation. This model has an entropic contribution that is the same as the combinatorial part of the UNIQUAC equation, due to differences in the size and shape of the 
molecules, and a residual contribution, due to energetic interactions between groups:

$$
\begin{gathered}
\ln \gamma_{i}=\ln \gamma_{i}^{S}+\ln \gamma_{i}^{G} \\
\ln \gamma_{i}^{S}=\ln \gamma_{i} \text { (comb) (UNIQUAC equation) } \\
\ln \gamma_{i}^{G}=\sum_{k=1}^{N G} v_{k i}\left(\ln \Gamma_{k}-\ln \Gamma_{k}^{(i)}\right)
\end{gathered}
$$

$\Gamma_{k}$ is the group residual activity coefficient and $\Gamma_{k}{ }^{(\prime)}$ is the residual activity coefficient of group $k$ in a reference solution containing only molecules of type $i$ :

$$
\begin{gathered}
\ln \Gamma_{k}=Q_{k}\left[1-\ln \left(\sum_{m=1}^{N G} \theta_{m} \psi_{m k}\right)-\sum_{m=1}^{N G} \frac{\theta_{m} \psi_{k m}}{\sum_{j=1}^{N G} \theta_{j} \psi_{j m}}\right. \\
\theta_{m}=\frac{Q_{m} X_{m}}{\sum_{j=1}^{N G} Q_{j} X_{j}} \\
X_{j}=\frac{\sum_{i=1}^{N} v_{j p} X_{i}}{\sum_{i=1}^{N} \sum_{m=1}^{N G} v_{m} X_{i}} \\
\psi_{m k}=\exp \left[-\frac{U_{m k}-U_{k k}}{R T}\right]=\exp \left[-\frac{a_{m k}}{T}\right]
\end{gathered}
$$

$X_{m}$ is the fraction of group $m$ in the mixture. The parameters $a_{m k}$ and $a_{k m}$ characterize the interactions between groups $m$ and $k$.

\section{Glossary}

A, B method of correlation of ternary data (Table VI)

$a_{\|} \quad$ UNIFAC group interaction parameter, $K$

$b_{y} \quad$ NRTL parameter, $\mathrm{K}$

$c_{l i} \quad$ UNIQUAC parameter, $\mathrm{K}$ $g_{l j}, u_{l j} \quad$ measure of the interaction energy between molecules $i$ and $j$

$F_{1}, F_{2}$ objective functions

$q_{1} \quad$ pure component area parameter of component $i$

$Q_{k} \quad$ group-area parameter for group $k$

$r_{i} \quad$ pure component volume parameter of component

$$
i
$$

$R_{k} \quad$ group-volume parameter of group $k$

$R \quad$ gas-law constant

$x_{i j} \quad$ molar fraction of component $i$ in phase $j$

$x_{i j} \quad$ weight fraction of component $i$ in phase $j$

$X_{k} \quad$ group fraction of group $k$

rmsd root-mean-square deviation

$z \quad$ lattice coordination number (equal to 10 )

\section{Greek Letters}

$\alpha_{\|} \quad$ nonaleatory NRTL parameter

$\gamma_{i}$ activity coefficient of component $i$

$\Gamma_{k} \quad$ activity coefficient of group $k$

$\Gamma_{k}(l) \quad$ activity coefficient of group $k$ in pure component $i$

$\phi_{i} \quad$ segment fraction of component $i$

$v_{k l} \quad$ number of groups of type $k$ in molecule $i$

$\vartheta_{1} \quad$ area fraction of component $i$

$\theta_{k} \quad$ area fraction of group $k$

$\tau_{i j} \quad$ NRTL of UNIQUAC parameter

$\psi_{n m} \quad$ UNIFAC parameter

Registry No. PFD, 306-94-5; 1-heptene, 592-76-7; $n$-heptane, 14282-5; $n$-hexane, 110-54-3.

\section{Llterature Clted}

(1) Bernardo-Gil, G. S.; Soares, L. J. S. J. Chem. Eng. Data 1987, 32 , 327.

(2) Soares, L. J. S.; Ashton, N. P.; Ellis, S. R. M. Proceedings of ISEC' 74 , Lyon, 1974; Vol. 2, p 1813.

(3) Renon, H.; Prausnitz, J. M. AIChE J. 1868, 14, 135

(4) Abrams, D. S.; Prausnitz, J. M. AIChE J. 1975, 21, 116.

(5) Fredensiund, A.; Jones, R. L.; Prausnitz, J. M. AIChE J. 1975, 21 1086.

(6) Bernardo-Gil, M. G.; Soares, L. J. S. Fluid Phase Equilib. 1986, 25. 291.

(7) Bondi, A. Physical Properties of Molecular Crystals, Liquids and Glasses; Wiley: New York, 1968.

Received for review March 28, 1988. Accepted October 12, 1988.

\title{
Estimation Procedures for Critical Constants
}

\author{
G. Raam Somayajulu \\ Thermodynamics Research Center, Texas A\&M University, College Station, Texas $77843-3111$
}

The procedures developed by Rledel, Lydersen, and Ambrose for the calculation of critical constants of organic compounds have been reexamined, and new procedures are proposed in terms of group Indices. These procedures are comblned with the procedures developed by Kreglewski for the calculation of critical constants of homologous series of compounds. The treatment has also been extended to Inorganic compounds.

\section{Introduction}

The knowledge of critical constants is important in the field of chemical process design in extrapolating, for example, the vapor pressures from the normal boiling point to the critical temperature (1). The procedures for the estimation of critical constants of organic compounds have been reviewed by several authors $(2-6)$. Among such procedures, the procedures developed by Lydersen (7), Ambrose (8-10), and Kreglewski $(11-13)$ have gained general recognition. They are noted for their simplicity and general accuracy. While the procedures developed by Lydersen and Ambrose yield satisfactory results for organic compounds of low molecular weight, the procedures developed by Kreglewski yield satisfactory results to homologous series of compounds, in general, of high molecular weight. We therefore propose to review these procedures and propose modified procedures for the calculation of critical constants. With this goal in mind we now introduce the following symbols. 